\title{
Dense arrays of nanopores as x-ray lithography masks
}

\author{
S. A. Knaack, J. Eddington, Q. Leonard, F. Cerrina, and M. Onellion ${ }^{\text {a) }}$ \\ Center for Nanotechnology, Physics and Electrical and Computer Engineering Departments, \\ University of Wisconsin, Madison, Wisconsin 53706
}

(Received 8 August 2003; accepted 19 February 2004)

\begin{abstract}
An anodized aluminum oxide nanopore has been used as an x-ray lithography mask to achieve a feature size of $\sim 35 \mathrm{~nm}$ on the polymethylmethacrylate photoresist. The mask was exposed using synchrotron radiation and demonstrates the feasibility of forming large arrays of regular nanostructures. () 2004 American Institute of Physics. [DOI: 10.1063/1.1705724]
\end{abstract}

Lithography comprises a worldwide effort to fabricate structures with the smallest possible feature sizes. Two types of lithography not using optical systems include contact and proximity; in the latter, there is a physical separation between the mask and photoresist. Masks are most often fabricated using electron beam lithography, a costly process requiring advanced and expensive equipment. It is also difficult to achieve large arrays of small features. Finally, it is difficult to achieve an extremely high packing density of features. In this letter we present results using a simple mask: a nanopore array of anodized aluminum oxide (AAO). ${ }^{1-3}$ Such a mask is fabricated by simple electrochemical means, ${ }^{4,5}$ and may have applications where arbitrary patterns are not needed (e.g., quantum dots). The x-ray mask has nanopore holes of $42 \pm 3.5 \mathrm{~nm}$, with an average hole-hole separation of $97 \pm 11 \mathrm{~nm}$. Interestingly, we obtained feature sizes of $35 \pm 3.8 \mathrm{~nm}$ on a polymethyl methacrylate (PMMA) photoresist. The image of the mask on the photoresist is not perfect over a large distance, but we show good transfer to the photoresist over lateral dimensions as large as $8 \times 8 \mathrm{~mm}^{2}$. The feature (hole) density is also notable, ${ }^{6,7} \sim 100 \mu \mathrm{m}^{-2}$. The significance of our results lies in achieving respectable Fresnel numbers ${ }^{8}$ (discussed below), and feature size and feature density using an inexpensive and readily manufactured mask.

The AAO mask thickness is $10.4 \pm 1.0 \mu \mathrm{m}$. We fabricated the mask to have this thickness, which is an optimum compromise between transmission and contrast. Experimentally, we have fabricated masks with pore diameters as small as $9 \pm 3 \mathrm{~nm},{ }^{9}$ and lateral areas as large as $\sim 1 \times 1 \mathrm{~cm}^{2}$. The specific conditions used include an electrochemical bath temperature of $1{ }^{\circ} \mathrm{C}$, a potential difference between anode and cathode of $40 \mathrm{~V}$, and an electrolytic solution of $0.3 \mathrm{M}$ oxalic acid.

To use the AAO mask in proximity lithography, we employed a 7- $\mu \mathrm{m}$-thick Kapton ${ }^{\mathrm{TM}}$ film that separated the mask from the PMMA. By holding the AAO mask on the Kapton, we could handle the fragile AAO mask and protect it from damage. The PMMA film was spin-coated onto a $\mathrm{Si}$ wafer. Following spin coating the PMMA was baked for $1 \mathrm{~min}$ at $160^{\circ} \mathrm{C}$. The PMMA thickness was $200 \mathrm{~nm}$, confirmed by both profilometer (inset of Fig. 1) and ellipsometer data. The inset of Fig. 1 shows a profilometer scan, in scale of mi-

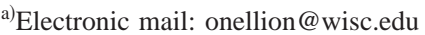

crometers, indicating the uniformity of the PMMA thickness. The AAO mask was secured with four drops of Duco Cement glue (Devcon Consumer Products) over the top AAO edge on the Kapton, leaving the mask flat on the surface of the Kapton.

The exposure was carried out on the ES1 beamline of the University of Wisconsin Synchrotron Radiation Center. ${ }^{10}$ It provides an x-ray light source with maximum intensity at a wavelength of $0.8 \mathrm{~nm}$. Initial exposures with doses of 1-5 $\mathrm{J} / \mathrm{cm}^{2}$ indicated an optimal dosage of $3 \mathrm{~J} / \mathrm{cm}^{2}$, with no observable features for doses of 1 and $2 \mathrm{~J} / \mathrm{cm}^{2}$ (underexposed), or for 4 and $5 \mathrm{~J} / \mathrm{cm}^{2}$ (overexposed). Subsequent exposures indicated almost the same features and contrasts for dosages between 2.5 and $3.5 \mathrm{~J} / \mathrm{cm}^{2}$. After exposure, the film was developed in a 1:3 (by volume) solution of methyl isobutyl ketone:isopropyl alcohol (IPA) for $90 \mathrm{sec}$, and rinsed in IPA for $2 \mathrm{~min}$.

We used a web applet ${ }^{11}$ from the Lawrence Berkeley Lab CXRO group to model the transmission rate through the aluminum oxide, and thus determine the contrast between the alumina and the open pores. The results suggest that at an average radiation wavelength of $0.8 \mathrm{~nm}(\sim 1550 \mathrm{eV})$ the transmission of $10.4 \mu \mathrm{m}$ of aluminum oxide is (0.03). We define $T_{\mathrm{CL}}$ as the transmission through the pore (which is taken to be 1), and $T_{\mathrm{ABS}}$ as the transmission through the alumina. The modulation is given as $M=\left(T_{\mathrm{CL}}\right.$

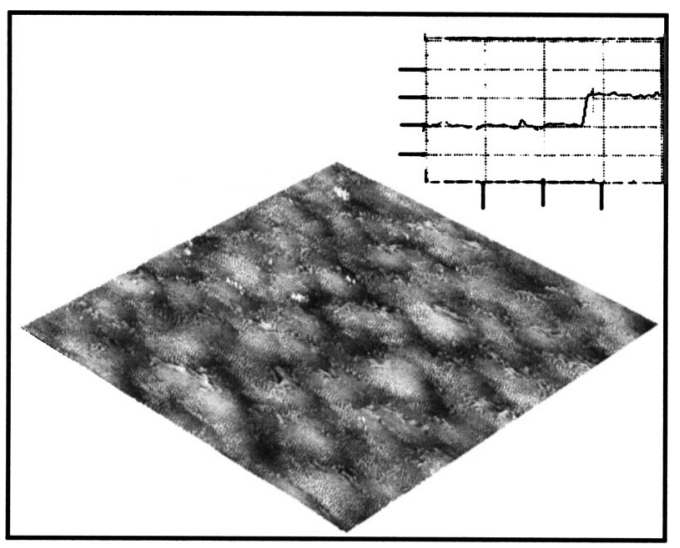

FIG. 1. An AFM three-dimensional perspective of a $500 \times 500 \mathrm{~nm}^{2}$ area of the exposed PMMA photoresist. Total vertical variation is $26 \mathrm{~nm}$. Inset: Profilometer data indicating a uniform thickness of $\sim 200 \mathrm{~nm}$ for the PMMA photoresist. The vertical scale is $200 \mathrm{~nm}$ per division, and the horizontal scale is $500 \mu \mathrm{m}$ per division. 
$\left.-T_{\mathrm{ABS}}\right) /\left(T_{\mathrm{CL}}+T_{\mathrm{ABS}}\right)$, and $R=T_{\mathrm{CL}} / T_{\mathrm{ABS}}$ for the optical contrast. Our modeling indicates a modulation of (0.94) and an optical contrast of (33.3).

The Fresnel number ${ }^{8}$ is defined as

$$
F=P^{2} / \lambda g
$$

where $P$ is the mask periodicity ( $97 \mathrm{~nm}$ here), $g$ the gap between the mask and the photoresist film $(7 \mu \mathrm{m}$ in this case), and $\lambda$ the average wavelength of the radiation $(0.8 \mathrm{~nm}$ in our work). The higher the Fresnel number, the better the expected contrast. The Fresnel number of our experimental setup is (1.68). It corresponds to a high level of contrast and a small feature size. ${ }^{8}$

Figure 1 provides a three-dimensional perspective of antiferromagnetic (AFM) results on the exposed photoresist. The data indicate a maximum (peak-to-trough) variation in the height of $26 \mathrm{~nm}$ over a $0.5 \times 0.5 \mu \mathrm{m}^{2}$ lateral area. By contrast, AFM measurements indicate the unexposed photoresist (PMMA) surface is quite flat, with only a $2.5 \mathrm{~nm}$ vertical variation over a $6.7 \times 9.3 \mu \mathrm{m}^{2}$ lateral area. ${ }^{12}$ The darker regions of the AFM image, which correspond to the lower vertical height, are the mask features (holes) transferred to the photoresist. Another indication of feature transfer is that the exposed PMMA shows more variation in height than unexposed PMMA, expected if there has been feature transfer.

Our results indicate a feature (hole) size of $35 \pm 3.8 \mathrm{~nm}$ in the exposure field. The area feature density is 100 features $\mu \mathrm{m}^{-2}$. Measuring the hole-hole distances yields an average of $97 \pm 13 \mathrm{~nm}$, identical within error to what was observed in the AAO mask.

Figure 2 shows scanning electron microscopy (SEM) images of the exposed PMMA field, and the AAO mask. Figure 2(a) is an SEM (Hitachi 1685) image of exposed PMMA at $20000 \times$ magnification. Features are not transferred over the entire field of the mask. Note the dotted area in the SEM image with few features. The upper left inset is an enlarged image of the PMMA features at $100000 \times$ magnification. The PMMA features are slightly smaller in diameter compared to the alumina nanopore mask, but do not exhibit six-fold symmetry as does the mask. The upper righthand-side inset shows a fast Fourier transform (FFT) analysis of the SEM image. It represents an analysis on a 256 $\times 256$ pixel square from the original $550 \times 475$ pixel image. Figure 2(b) shows a SEM (LEO model $1540 \mathrm{VP}$ ) image of the mask at a magnification comparable to that of the exposed PMMA image, 16650×. Here, the long-range order and uniform feature (hole) spacing is evident. The upper lefthand-side inset of Fig. 2(b) shows the individual AAO pores of the $\mathrm{x}$-ray mask. The upper right-hand-side inset is a FFT analysis on a center $256 \times 256$ pixel square of the original $1024 \times 768$ pixel image. We thus performed FFT analysis of both the mask and the PMMA photoresist image for comparison.

The AAO mask FFT exhibits a well-defined center ring feature, which indicates long-range as well as short-range pore-pore order. This is in contrast to the solid circular form as the main feature of the exposed PMMA FFT, which suggests short-range but not long-range, feature-feature order. The diameters of the solid circle in the PMMA FFT and ring Downloaded 24 Feb 2007 to 128.104.198.190. Redistribution subject to AlP license or copyright, see http://apl.aip.org/apl/copyright.jsp
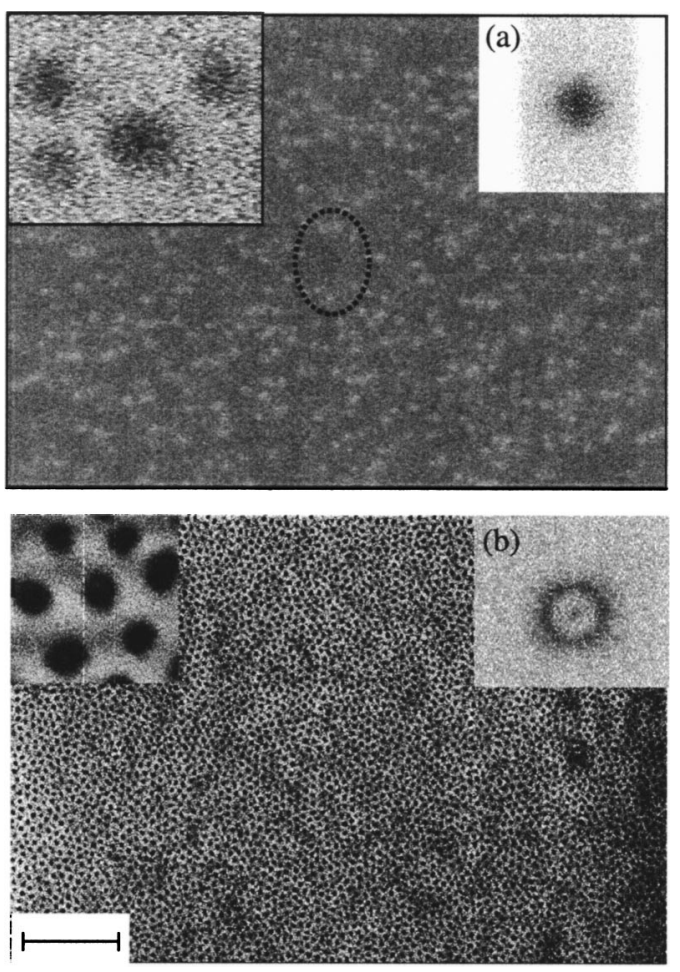

FIG. 2. (a) SEM image of exposed photoresist $(20000 \times)$. Dotted area is an example of areas containing few lithographic features. Upper left-hand-side inset: A $100000 \times$ SEM image of the exposure features. Upper right-handside inset: FFT result for $256 \times 256$ pixel square. (Original image size: 550 $\times 475$ pixels.) (b) SEM image of AAO mask $(16630 \times)$. Bar scale on the lower left-hand side is $1.0 \mu \mathrm{m}$. Upper left-hand-side inset: SEM image of AAO mask (191200×) showing individual pores and hexagonal order. Upper right-hand-side inset: FFT result for $256 \times 256$ pixel square. (Original image size: $1024 \times 768$ pixels.)

in the mask FFT are within $6 \%$ of each other, consistent with the pore-pore and feature-feature distances measured directly on the SEM micrographs. Our interpretation of FFT data follows Ref. 13.

There are two possible explanations for the incomplete transfer of features in some areas. Some of the AAO mask pores could be blocked by remnant sections of the AAO mask barrier layer. Another possibility is that the pores in some areas may not have been sufficiently parallel, allowing absorption and scattering of $\mathrm{x}$ rays in the mask. Recall that the mask aspect ratio (pore length/diameter) is $\sim 240$, so even a small variation in angular alignment would change the transmission.

In summary, we have reported three significant results: (1) The density of $35 \mathrm{~nm}$ hole features $\left(\sim 100 \mu \mathrm{m}^{-2}\right)$ is greater than previous reports. ${ }^{6,7}$ (2) Obtaining a comparatively high Fresnel number, easily reached in our conditions, makes the AAO mask technique seem very promising. (3) We have obtained small $(\sim 35 \mathrm{~nm})$ features in proximity lithography using a low-technology, inexpensive method for fabricating x-ray masks.

The optical and FFT analysis of the exposed photoresist indicates that the exposure is imperfect, but that short-range order and large-area exposure have been achieved. Future work should include improving how close to perfectly ordered, long-lateral-range transfer we can achieve by better AAO fabrication and alignment control of the mask with respect to the X-ray beam. Because AAO nanopores with 
pore diameters as small as $\sim 7 \mathrm{~nm}$ have been reported, ${ }^{8}$ this approach seems promising as $\mathrm{x}$-ray lithography continues to strive to reach feature sizes well below $50 \mathrm{~nm}$.

The authors would like to thank S. Liu for valuable discussion and comments, funding and facilities use from the Center for Nano-Technology, and the Synchrotron Radiation Center (both of the University of Wisconsin-Madison) for facilities use. The SRC is funded in party by NSF grant No. DMR-0084402 S.A.K. would like to thank the Undergraduate Hilldale Fellowship Program of the University of Wisconsin-Madison for funding. This work was performed as part of S.A.K.'s undergraduate research experience.

${ }^{1}$ H. Masuda and K. Fukuda, Science 268, 1466 (1995).

${ }^{2}$ O. Jessensky, F. Müller, and U. Gösele, Appl. Phys. Lett. 72, 1173 (1998).

${ }^{3}$ K. Nielsch, J. Choi, K. Schwirn, R. B. Wehrspohn, and U. Gösele, Nano Lett. 2, 677 (2002).
${ }^{4}$ A. P. Li, F. Müller, A. Birner, K. Nielsch, and U. Gösele, J. Appl. Phys. 84, 6023 (1998).

${ }^{5}$ O. Jessensky, F. Müller, and U. Gösele, J. Electrochem. Soc. 145, 3735 (1998).

${ }^{6}$ J. O. Choi, A. I. Akinwande, and H. I. Smith, J. Vac. Sci. Technol. B 19, 900 (2001).

${ }^{7}$ C. A. Ross, H. I. Smith, T. Savas, M. Schattenberg, M. Farhoud, M. Hwang, M. Walsh, M. C. Abraham, and R. J. Ram, J. Vac. Sci. Technol. B 17, 3168 (1999).

${ }^{8}$ M. Kahn, G. Han, G. Tsvid, T. Kitayama, J. Maldonado, and F. Cerrina, J. Vac. Sci. Technol. B 19, 2423 (2001).

${ }^{9}$ Z. Zhang, D. Gekhtman, M. S. Dreselhaus, and J. Y. Ying, Chem. Mater. 11, 1659 (1999).

${ }^{10}$ R. Cole, P. Anderson, G. M. Wells, E. Brodsky, K. Yamazaki, and F. Cerrina, Proc. SPIE 1671, 461 (1992).

${ }^{11} \mathrm{http} / / /$ www-cxro.lbl.gov/optical_constants/Found under x-ray transmission: of a solid.

${ }^{12} \mathrm{~S}$. Liu, CNTech (private communication).

${ }^{13}$ S. Shingubara, O. Okino, Y. Sayama, H. Sakaue, and T. Takahagi, Jpn. J. Appl. Phys. 36, 7791 (1997). 